\title{
Deformation and failure of bulk metallic glasses under different initial temperatures
}

\author{
J.C. $\mathrm{Li}^{1}$, X.W. Chen ${ }^{1, \mathrm{a}}$, and F.L. Huang ${ }^{2}$ \\ ${ }^{1}$ Institute of Structural Mechanics, China Academy of Engineering Physics, Mianyang, Sichuan 621900, China \\ ${ }^{2}$ State Key Laboratory of Explosion Science and Technology, Beijing Institute of Technology, Beijing 100081, China
}

\begin{abstract}
Based on the coupled thermo-mechanical model, a constitutive model for bulk metallic glasses (BMGs), which is generalized to the multi-axial stress state and considers the effects of free volume, heat and hydrostatic stress, has been modified in the present paper. Besides, a failure criterion of critical free volume concentration is introduced based on the coalescence mechanism of free volume. The constitutive model as well as the failure criterion is implemented into the LS-DYNA commercial software by user material subroutine (UMAT). Then FEM simulations for different initial material temperatures are conducted and the evolutions of material parameter as well as corresponding macroscopic mechanical behaviour of material are analyzed. Relative analysis shows that the initial material temperature significantly affects the deformation and failure of material.
\end{abstract}

\section{Introduction}

Bulk metallic glasses (BMGs) have many excellent mechanical and physical performances. It usually show good plasticity even superplasticity while under the condition of high temperature (within the supercooled liquid region) and low strain rate [1-3]. However, in the case of low temperature (below the glass transition temperature $T_{g}$ ) and high stress or high strain rate, seriously localized shear bands are easily to be produced during the deformation process. Fracture occurs immediately along the propagation of shear bands and thus results in a catastrophic failure phenomenon. Moreover, the material usually shows an asymmetry between tension and compression [4,5]. All these particular properties promote that BMGs may have many engineering applications, e.g., the good plasticity is beneficial for the precise microforming process [6], and the localized shear bands may be employed in the material optimization of kinetic energy penetrator (KEP) $[7,8]$.

Related analyses demonstrated that the microstructure of material may have an important effect on the shear banding process in BMGs [9-11]. Besides, it is usually found a significant temperature rise in the fracture surface [5]. Presently, a coupled thermo-mechanical shear model [5,11], deriving from the free volume model and the adiabatic deformation theory, could predict the shear banding behaviour of BMGs at room temperature relatively well. Comparatively, for the homogeneous flow of BMGs at high temperature $\left(T \geq T_{g}\right)$, the related analyses usually follow the models used for the crystalline alloys $[1,3]$. Regarding the yielding and failure of BMGs,

${ }^{a}$ Corresponding author: chenxiaoweintu@yahoo.com some macroscopic failure criteria have been suggested and successfully describe the deviation of fracture surface from the maximum shear stress plane $[12,13]$. However, the micro-mechanism of deformation and failure of BMGs is still under the exploration process [14-17]. Recently, the simulation based on finite element method (FEM) is gradually employed for investigating the mechanical behavior of BMGs. Related work includes the theoretical constitutive modeling and the construction technique of the geometric model, etc. $[18,19]$.

Integrating with related investigations, the present manuscript further generalizes the coupled thermomechanical shear model into the multi-axial stress status, and takes all the contributions of free volume, temperature and hydrostatic stress into account. Besides, based on the coalescence mechanism of free volume in BMGs, a failure criterion of critical free volume concentration, $\xi_{c}$, is introduced. Furthermore, the generalized multiaxial-stress-status model is extended to describe the mechanical behavior of BMGs at high temperature $(T \geq$ $T_{g}$ ) by estimating related material parameters. Then the constitutive model and failure criterion are implemented into LS-DYNA commercial software through the user material subroutine (UMAT), and FEM simulations for different initial material temperatures are conducted integrated with the related test data. The evolutions of parameters as well as their effects on the mechanical behavior of material are analyzed, and corresponding mechanism of the macroscopic behavior is discussed. Related analyses show that the initial temperature has a significant effect on the deformation and failure of BMGs. Especially, the microstructure as well as corresponding mechanical behavior of material is extremely different while the initial temperature is below or within the supercooled liquid region. 


\section{Constitutive model}

\subsection{Constitutive equations and failure criterion}

Basing on the free volume model, Dai et al. considered the effect of temperature additionally and suggested a coupled thermo-mechanical model for BMGs $[5,11]$. However, the model is still limited within the one-dimensional simple shear mode. Gao [18] generalized the one-dimensional free volume model into the multi-axial stress status but ignoring the effect of temperature. Actually, during the deformation process of BMGs, the hydrostatic stress (or the normal stress acting on the shear plane), which derived from the shear-induced dilatation of material, also contributes to the deformation and failure $[4,13,17]$. Thus, the effect of hydrostatic stress should be considered in the constitutive model. By further considering the effect of hydrostatic stress on the deformation, the evolution of free volume and temperature, a modified thermo-mechanical model for BMGs which is generalized to multi-axial stress status could be obtained:

$$
\begin{gathered}
\sigma_{e}^{\prime}=\Lambda \sigma_{m}+\sqrt{J_{2}} \\
\dot{\varepsilon}_{i j}=\dot{\varepsilon}_{i j}^{e}+\dot{\varepsilon}_{i j}^{p} \\
\dot{\varepsilon}_{i j}^{e}=\frac{1+v}{E}\left(\dot{\sigma}_{i j}-\frac{v}{1+v} \dot{\sigma}_{k k} \delta_{i j}\right) \\
\dot{\varepsilon}_{i j}^{p}=f \exp \left(-\frac{\Delta G^{m}}{K_{B} T}\right) \exp \left(-\frac{1}{\xi}\right) \sinh \left(\frac{\sigma_{e}^{\prime} \Omega}{2 K_{B} T}\right) \frac{S_{i j}}{\sigma_{e}} \\
\dot{\xi}=D \nabla^{2} \xi+\frac{1}{\alpha} f \exp \left(-\frac{\Delta G^{m}}{\sigma_{B} T}\right) \exp \left(-\frac{1}{\xi}\right) \\
\times\left\{\frac { 2 K _ { B } T } { \xi v ^ { * } s } \left[\begin{array}{c}
\sigma_{e}^{\prime} \\
\left.\left.\cosh \left(\frac{\sigma_{e}^{\prime} \Omega}{2 K_{B} T}\right)-1\right]-\frac{1}{n_{D}}\right\} \\
\dot{T}=\kappa \nabla^{2} T+\frac{\beta_{T Q}}{\rho C_{v}} \sigma_{e}^{\prime} \dot{\varepsilon}_{e}^{p}
\end{array}\right.\right.
\end{gathered}
$$

In Eqs. (1)-(3), a dot over a quantity () denotes differentiation with time.

Equation (1) implies the material is assumed to be elastically isotropic, and the strain is decoupled into the elastic and plastic parts. $E$ and $v$ are the Young's modulus and Poisson's ratio, respectively; $f$ is the frequency of atomic vibration and usually adopts the Debye frequency; $\Delta G^{m}$ is the activation energy; $K_{B}$ is the Boltzmann constant and $T$ the absolute temperature; $\xi=\bar{v}_{f} /\left(\alpha v^{*}\right)$ is the free volume concentration in BMGs (here $\bar{v}_{f}, v^{*}$ and $\alpha$ are, respectively, the average free volume per atom, critical volume (hard-sphere volume of an atom) and a geometrical factor) [10]; $\Omega$ is the average atomic volume; $\sigma_{e}$ is the vonMises effective stress, in which $J_{2}=\sqrt{S_{i j} S_{i j} / 2}$ is the $J_{2}$ invariant of stress, $S_{i j}=\sigma_{i j}-\delta_{i j} \sigma_{m}$ is the deviatoric stress tensor and $\sigma_{m}=\sigma_{k k} / 3$ the mean stress; $\sigma_{e}^{\prime}$ is an effective stress which considers the contribution of hydrostatic stress, in which $\Lambda$ is the hydrostatic stress sensitivity factor.

Equation (2) is the free volume evolution equation, in which $D$ is the diffusion coefficient of free volume; $s=$ $E /[3(1-v)]$ is the Eshelby modulus and $n_{D}$ the number of diffusive jumps necessary to annihilate a free volume as $v^{*}$. Equation (2) indicates there are three different physical processes that alter the local free volume concentration: diffusion (the first term), generation induced by stresses (the first term in bracket) and annihilation by the atomic rearrangement (the second term in bracket).

Equation (3) is the temperature evolution equation, where $\kappa=K /\left(\rho C_{v}\right)$ is the thermal diffusivity (here $K$, $\rho$ and $C_{v}$ are the thermal conductivity, mass density and specific heat at constant volume, respectively); $\varepsilon_{e}^{p}=$ $\sqrt{2 \varepsilon_{i j}^{p} \varepsilon_{i j}^{p} / 3}$ is the effective plastic strain. $\beta_{T Q}$ is the Taylor-Quinney coefficient which is usually taken as 0.9 or considered to be related to the strain rate. Since the adiabatic deformation usually occurs in the case of high strain rate, the Taylor-Quinney coefficient $\beta_{T Q}$ may be defined as a function of irreversible part of the effective plastic strain rate [11]:

$$
\beta_{T Q}=\frac{1}{\pi} \arctan \left(\frac{1}{3} \tan \frac{2 \pi}{5} 1 g \frac{\dot{\varepsilon}_{e}^{p}}{\dot{\varepsilon}_{0}}\right)+\frac{1}{2}
$$

in which $\dot{\varepsilon}_{0}$ is the referenced strain rate and takes a value of $1 \mathrm{~s}-1$. Note from Eq. (4) that it satisfies $\lim _{\varepsilon_{e}^{p} \rightarrow 0} \beta_{T Q}=$ 0 and $\lim _{\varepsilon_{e}^{\dot{p}} \rightarrow \infty} \beta_{T Q}=1$. Moreover, Zhou et al. [20]'s analysis demonstrated that when the material yields, the stress located within the shear band begins to collapse and unloads its neighboring material. Then the unloading wave propagates towards the neighboring zone of the shear band. When failure occurs in the shear band, the deformation energy in the neighboring zone where the unloading wave has arrived will release on the fracture surface and induces extreme temperature rise. Thus, in the simulation we could further modify the coefficient $\beta_{T Q}$ when the material is going to fail.

Furthermore, different from crystalline alloys, BMGs display a few special failure properties. The related macroscopic failure criteria are difficult to describe the failure mechanism completely. Researchers analyzed the failure process based on the change of microstructure of BMGs $[15,16]$, and demonstrated that the free volume will be created during the deformation process and then coalesce together. Micro-voids will be formed due to the coalescence of free volumes and further leads the damage and failure eventually. Thus, the failure criterion in the present model will be based on the evolution of microstructure. It is assumed that failure occurs when the net free volumes exceed a critical value, i.e. corresponding failure criterion is that the free volume concentration $\xi$ achieves the critical value $\xi_{c}$ :

$$
\xi \geq \xi_{c}
$$


Table 1. Mechanical properties and parameters of $\mathrm{Zr}_{41.25} \mathrm{Ti}_{13.75} \mathrm{Cu}_{12.5} \mathrm{Ni}_{1} 0 \mathrm{Be}_{22.5}$ (Vitreloy 1) BMG.

\begin{tabular}{|c|c|c|c|}
\hline Parameter & Notation & Unit & Value \\
\hline \multirow{2}{*}{ Young's modulus } & \multirow{2}{*}{$E$} & \multirow[b]{2}{*}{$\mathrm{GPa}$} & 96 for $T_{0}<T_{g}$ \\
\hline & & & $E\left(T_{0}, \dot{\varepsilon}\right)$ for $T_{0} \geq T_{g}$ \\
\hline Poisson ratio & $v$ & - & 0.36 \\
\hline Glass transition temperature & $T_{g}$ & $\mathrm{~K}$ & 625 \\
\hline Melting temperature & $T_{m}$ & $\mathrm{~K}$ & 993 \\
\hline Density & $\rho$ & $\mathrm{kg} / \mathrm{m}^{3}$ & 6125 \\
\hline Specific heat at constant volume & $C_{v}$ & $\mathrm{~J} /(\mathrm{kg} \cdot \mathrm{K})$ & 400 \\
\hline Frequency of atomic vibration & $f$ & $\mathrm{~s}^{-1}$ & $1 \times 10^{13}$ \\
\hline Average atomic volume & $\Omega$ & $\mathrm{A}^{3}$ & 25 \\
\hline Critical volume & $v^{*}$ & $\mathrm{~A}^{3}$ & 20 \\
\hline Initial temperature & $T_{0}$ & $\mathrm{~K}$ & $295-683$ \\
\hline Initial free volume concentration & $\xi_{0}$ & - & 0.05 \\
\hline Critical free volume concentration & $\xi_{c}$ & - & 0.065 \\
\hline \multirow{2}{*}{ Geometrical factor } & \multirow{2}{*}{$\alpha$} & \multirow[b]{2}{*}{ - } & 0.05 for $T_{0}<T_{g}$ \\
\hline & & & $\alpha\left(T_{0}\right)$ for $T_{0} \geq T_{g}$ \\
\hline Activation energy & $\Delta G^{m}$ & $\mathrm{eV}$ & $\Delta G^{m}(\dot{\varepsilon})$ \\
\hline Number of diffusive jumps & $n_{D}$ & - & $n_{D}\left(T_{0}\right)$ \\
\hline Hydrostatic stress sensitivity factor & $\Lambda$ & - & $\Lambda_{C}=0.05$ \\
\hline
\end{tabular}

\subsection{Finite element implementation of constitutive model}

Through the UMAT subroutine, the above constitutive model and the failure criterion are implemented into the LS-DYNA commercial software. As mentioned in [18], if the thermal conduction and the diffusion of free volume are both considered, it will be difficult to match up the two different length scales in the FEM simulation. As the influences of the diffusion of free volume and the thermal conduction are small $[10,11]$, to avoid the trouble of matching up the different length scales, the two diffusion issues are ignored in the FEM implementation. After such a predigesting treatment, the corresponding FEM simulation is independent on the length scale.

\subsection{Estimation of material parameters}

For convenience of comparative analysis with the corresponding test data, the typical $\mathrm{Zr}_{41.2} \mathrm{Ti}_{13.8} \mathrm{Cu}_{12.5} \mathrm{Ni}_{10.0} \mathrm{Be}_{22.5}$ (Vitreloy 1) BMG is chosen as the model material. Integrated with the related literatures $[10,11,15,18,19]$, corresponding material parameters are listed in Table 1. The authors had conducted a detailed analysis on the material parameters, and the related discussions could be referred in [21].

\section{Deformation and failure of BMGs under different initial temperatures}

In order to validate the applicability of the constitutive model, FEM simulations will be conducted and also the effect of various parameters on the mechanical behavior of BMGs will be analyzed. For the computational convenience, a one-element geometrical model is adopted. As the characteristic size of the test sample is usually in millimeter scale, we choose a hexahedral element with side length of $1 \mathrm{~mm}$.

The mechanical properties of BMGs under different strain rates and temperatures varies significantly. In the following he evolutions of various material parameters at different initial temperatures will be analyzed in detail, especially the inner variables. Then corresponding mechanism of the macroscopic mechanical behavior is discussed based on the parameter analysis. In the simulations the failure of material will be ignored for observing the evolutions of parameters thoroughly and discussing their influences. Besides, the analysis mainly focuses on the case of uni-axial compression, and deformation and failure characteristics of BMGs under tension or shear are similar to that under compression.

For convenience to compare with corresponding test data, two typical strain rates, $\dot{\varepsilon}=10^{-1} \mathrm{~s}^{-1}$ and $10^{3} \mathrm{~s}^{-1}$, are selected. The evolutions of various parameters under different initial temperatures are shown in Figs. 1-2, respectively, in which the abscissa represents the engineering strain. Besides, the test data are symbolized as "test" and indicate the results in [3]. The data with no special symbol represent the simulation results. Same symbols will be used in the latter.

It can be seen in Fig. 1(a) the simulation results agree with the test data well. The yielding stress $\sigma_{Y}$ decreases gradually with the increase of initial temperature, and the reduction is much more significant after $T_{0} \geq T_{g}$. Besides, within the supercooled liquid region, the softening matter becomes slower and the collapse of stress after softening decreases gradually with increasing the initial temperature. Almost no softening matter occurs at $683 \mathrm{~K}$. It further confirms that BMGs within the supercooled liquid region are suitable for precise microforming, and it can be inferred that a slight increase of material temperature will lead a significant reduction of difficulty in microforming process.

The evolution of temperature $T$ is similar to that of $\varepsilon_{e}^{p}$ in Fig. (1c). However, due to the strain rate dependence of the Taylor-Quinney coefficient $\beta_{T Q}$ in Eq. (4), the temperature rises $\Delta T$ under various initial temperatures are all relatively low. It deviates from the experimental results as the fracture surface of BMGs usually shows a heat phenomenon. The possible reason is that, the 


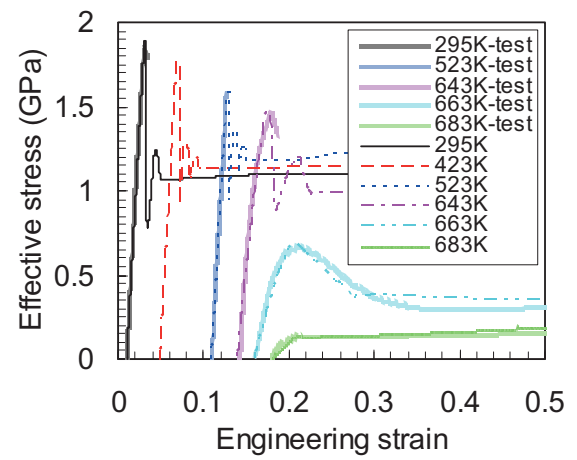

(a) Effective stress

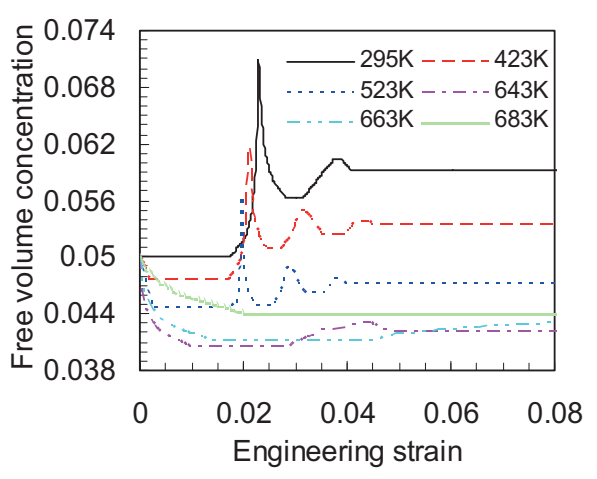

(b) Free volume concentration

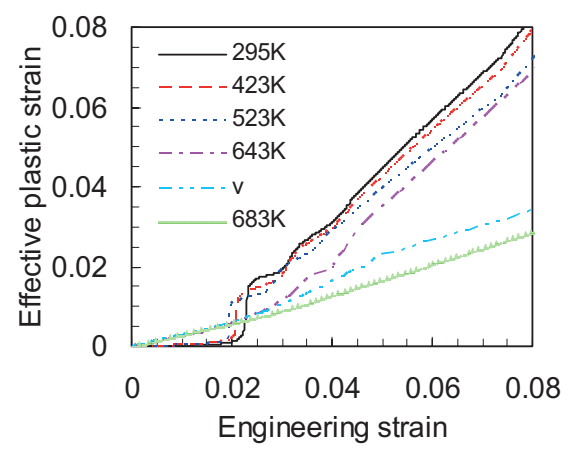

(c) Effective plastic strain

Figure 1. Evolutions of parameters for uniaxial compression at $\dot{\varepsilon}=10^{-1} \mathrm{~s}^{-1}$ and various initial temperatures.

simulations only consider the deformation energy of one element, whilst the neighboring deformation energy also releases on the fracture surface and induce significant temperature rise for the actual material [20]. When the simulation considers the failure of $\mathrm{BMG}$, we can change the value of $\beta_{T Q}$ and correspondingly $\Delta T$ is modified. Related analysis is seen in [21].

By comparing Fig. 2 with Fig. 1 it can be found that while $T_{0}<T_{g}$, the evolutions of parameters are similar to each other for the two strain rates. The yielding stress and strain increase slightly for the higher strain rate, and the values of $\xi_{\text {peak }}$ as well as $\varepsilon_{e}^{p}$ after the precipitous increase also have a slight increase, i.e. deformation localization becomes more significant. Comparatively, at

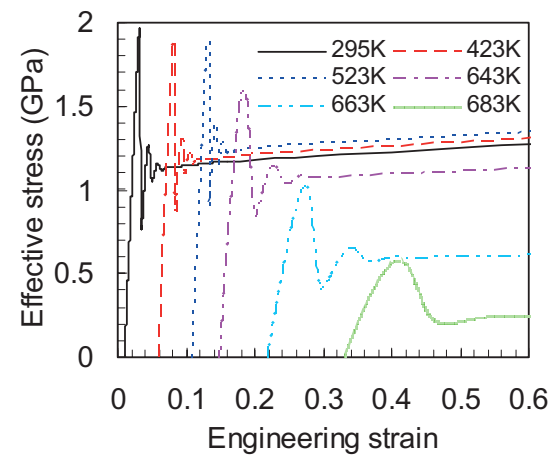

(a) Effective stress

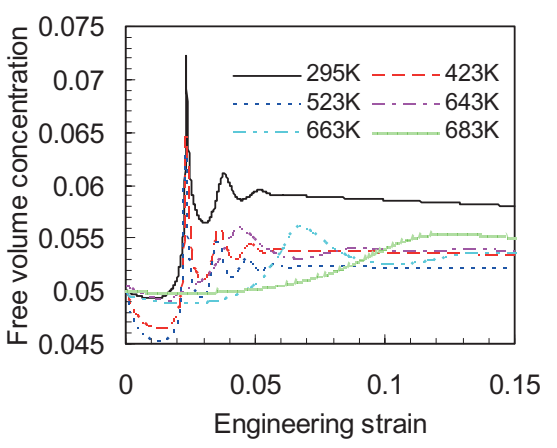

(b) Free volume concentration

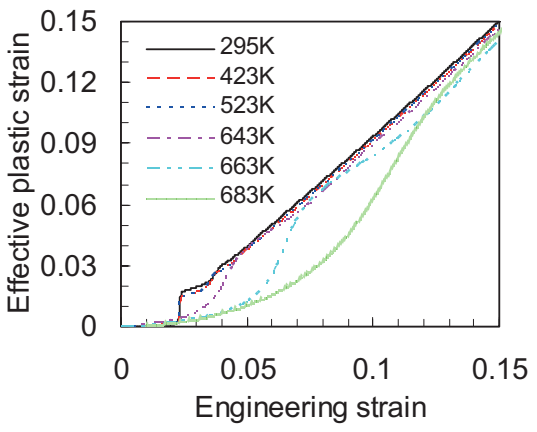

(c) Effective plastic strain

Figure 2. Evolutions of parameters for uniaxial compression at $\dot{\varepsilon}=10^{3} \mathrm{~s}^{-1}$ and various initial temperatures.

the temperatures of $T_{0} \geq T_{g}$, the differences between evolutions under the two strain rates are distinct. While under high strain rate, the yielding stress and strain increase distinctly, and the softening of stress becomes much more significant. Moreover, the value of $\xi$ at the latter stage exceeds the initial value $\xi_{0}$, and $\varepsilon_{e}^{p}$ exhibits a certain precipitous increase characteristic. These properties indicate that though within the supercooled liquid region, the inner structure of material will become more disordered after deformation under high strain rates, and deformation localization occurs, i.e. the homogeneous flow begin to translate into inhomogeneous deformation. It accords with the actual mechanical behavior in the tests [3].

To further investigate the deformation and failure characteristics, the variations of peak free volume 


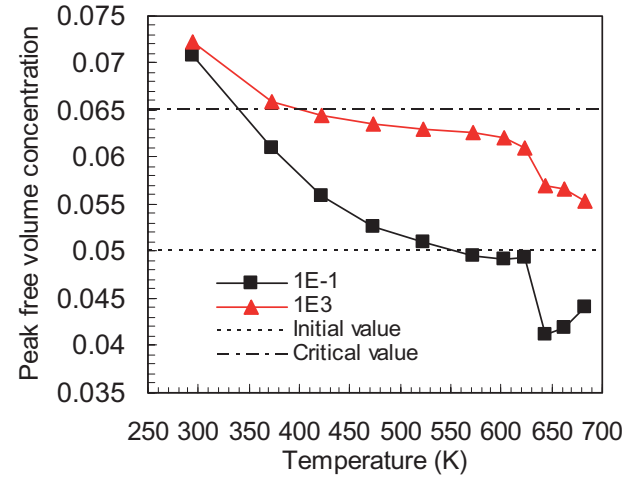

Figure 3. Variations of peak free volume concentration $\xi_{\text {peak }}$ via the initial temperature at different strain rates.

concentration $\xi_{\text {peak }}$ via the initial temperatures at the two strain rates are listed in Fig. 3. As $\xi$ no longer changes after decreasing to a certain value at $\dot{\varepsilon}=10^{-1} \mathrm{~s}^{-1}$ and $T_{0} \geq T_{g}$ (see Fig. 1(b)), the stable value is adopted as the value of $\xi_{\text {peak }}$.

As shown in Fig. 3, $\xi_{\text {peak }}$ decreases with increasing the initial temperature, and the decrease is more speedy for a lower strain rate. Besides, in the case of $\dot{\varepsilon}=$ $10^{-1} \mathrm{~s}^{-1}, \xi_{\text {peak }}$ is smaller than $\xi_{0}$ when $T_{0} \geq 550 \mathrm{~K}$, implying that the deformation of material may display as a homogeneous flow even if it hasn't been within the supercooled liquid region. Being as the test evidence, Nieh et al. [2] demonstrated when $T_{0}$ increases up to a value of $0.7 \mathrm{~T}_{g}$, the BMG deformation indeed displayed as a homogeneous flow. In contrast, in the case of $\dot{\varepsilon}=$ $10^{3} \mathrm{~s}^{-1}, \xi_{\text {peak }}$ is significantly larger than $\xi_{0}$, indicating the inner structure of material becomes more disordered after deformation, and deformation localization occurs, i.e., the homogeneous flow translates into inhomogeneous deformation. However, the value of $\xi_{\text {peak }}$ is still less than that of $\xi_{c}$ when $T_{0} \geq 423 \mathrm{~K}$, which indicates the onset of failure is relatively more difficult for a higher initial temperature.

\section{Conclusions}

Based on the coupled thermo-mechanical model, a multiaxial-stress-status constitutive model which considers the effects of the free volume, temperature and hydrostatic stress on the deformation and failure of BMGs is further suggested in the present manuscript. Besides, a failure criterion of critical free volume concentration is introduced. The constitutive model and the failure criterion are implemented into the LS-DYNA commercial software through UMAT subroutine. The deformation and failure characteristics of BMGs under different initial temperatures are analyzed based on the FEM simulations.

Related analysis shows that the initial temperature significantly affects the mechanical behavior of BMGs. While under the condition of $T_{0}<T_{g}$, the material displays a precipitous softening of stress after yielding, and the deformation and failure process is very speedy and highly localized. In contrast, within the supercooled liquid region $\left(T_{0} \geq T_{g}\right)$, the softening character of material is weakened and the material prefers to flow homogeneously. However, the deformation will translate into inhomogeneous status if distinctly increasing the strain rate. Generally, when the initial temperature increases, the deformation localization character is weakened and failure is more difficult to occur in BMGs.

The project is supported by the National Natural Science Foundation of China-NSAF (10976100), the National Outstanding Young Scientists Foundation of China (11225213) and the National Natural Science Foundation of China (11221202).

\section{References}

[1] Inoue A., Kawamura Y., Shibata T., et al. Viscous flow deformation in supercooled liquid state of bulk amorphous $\mathrm{Zr}_{5} 5 \mathrm{Al}_{1} 0 \mathrm{Ni}_{5} \mathrm{Cu}_{3} 0$ alloy. Materials Transactions JIM, 1996, 39 (6): 1337-1341.

[2] Nieh T.G., Wadsworth J., Liu C.T., et al. Plasticity and structural instability in a bulk metallic glass deformed in the supercooled liquid region. Acta Materialia, 2001, 49; 15: 2887-2896.

[3] Lu J., Ravichandran G., Johnson W.L. Deformation behavior of the $\mathrm{Zr}_{41.2} \mathrm{Ti}_{13.8} \mathrm{Cu}_{12.5} \mathrm{Ni}_{10} \mathrm{Be}_{22.5}$ bulk metallic glass over a wide range of strainrates and temperatures. Acta Materialia, 2003, 51: 3429-3443.

[4] Zhang Z.F., Eckert J., Schultz L. Difference in compressive and tensile fracture mechanisms of $\mathrm{Zr}_{59} \mathrm{Cu}_{20} \mathrm{Al}_{10} \mathrm{Ni}_{8} \mathrm{Ti}_{3}$ bulk metallic glass. Acta Materialia, 2003, 51:1167-1179.

[5] Dai L.H., Bai Y.L. Basic mechanical behaviors and mechanics of shear banding in BMGs. International Journal of Impact Engineering, 2008, 35: 704-716.

[6] Nishiyama N., Inoue A. Glass transition behavior and viscous flow working of $\mathrm{Pd}_{40} \mathrm{Cu}_{30} \mathrm{Ni}_{10} \mathrm{P}_{20}$ amoephous alloy. Materials Transactions JIM, 1999; 40 (1): 64-70.

[7] Conner R.D., Dandliker R.B., Scruggs V., et al. Dynamic deformation behavior of tungsten-fiber / metallic-glass matrix composites. International Journal of Impact Engineering. 2000; 24: 435-444.

[8] Choi-Yim H., Conner R.D., Szuecs F., et al. Quasistatic and dynamic deformation of tungsten reinforced $\mathrm{Zr}_{5} 7 \mathrm{Nb}_{5} \mathrm{Al}_{1} 0 \mathrm{Cu}_{15.4} \mathrm{Ni}_{12.6}$ bulk metallic glass matrix. Scripta Materialia, 2001; 45: 10391045.

[9] Spaepen F. A microscopic mechanism for steady state inhomogeneous flow in metallic glasses. Acta Metallurgica, 1977, 25 (9): 407-415.

[10] Huang R., Suo Z., Prevost J.H. Inhomogeneous deformation in metallic glasses. Journal of the Mechanics and Physics of Solids, 2002, 50: 10111027.

[11] Jiang M.Q., Dai L.H. On the origin of shear banding instability in metallic glasses. Journal of the Mechanics and Physics of Solids, 2009, 57: 12671292.

[12] Zhang Z.F., Eckert J. Unified Tensile Fracture Criterion. Physical Review Letters, 2005, PRL 94: 094301-1-4. 
[13] Chen Y., Jiang M.Q., Wei Y.J., et al. Failure criterion for metallic glasses. Philosophical Magazine, 2011, 91(36): 4536-4554.

[14] Zhang Z.F., Wu F.F., Gao W., et al. Wavy cleavage fracture of bulk metallic glass. Applied Physics Letters, 2006, 89: 251917-1-3.

[15] Wright W.J., Hufnagel T.C., Nix W.D. Free volume coalescence and void formation in shear bands in metallic glass. Journal of Applied Physics, 2003; 93 (8):1432-1437.

[16] Xue Y.F., Cai H.N., Wang L., et al. Effect of loading rate on failure in Zr-based bulk metallic glass. Materials Science and Engineering A, 2008, 473: $105-110$.

[17] Jiang M.Q., Ling Z., Meng J.X., et al. Energy dissipation in fracture of bulk metallic glasses via inherent competition between local softening and quasicleavage. Philosophical Magazine, 2008, 21(8): 407-426.
[18] Gao Y.F. An implicit finite element method for simulating inhomogeneous deformation and shear bands of amorphous alloys based on the free-volume model. Modelling and Simulation in Materials Science and Engineering, 2006, 14: 1329-1345.

[19] Chen Y., Jiang M.Q., Dai L.H. How does the initial free volume distribution affect shear band formation in metallic glass?. Science China: Physics, Mechanics \& Astronomy. 2011, 54 (8): 1488-1494.

[20] Zhou F., Wright T.W., Ramesh K.T. A numerical methodology for investigating the formation of adiabatic shear bands. Journal of the Mechanics and Physics of Solids, 2006, 54: 904-926.

[21] Li J.C., Wei Q., Chen X.W., et al. On the mechanism of deformation and failure in bulk metallic glasses. Materials Science and Engineering A, 2014, 610: 91 105. 